\title{
A COOCORRÊNCIA DE PLANTAS NA CAATINGA PODE SER EXPLICADA PELO PROCES- SO DE FACILITAÇÃO? ESTUDO DE CASO COM DUAS ESPÉCIES DE FABACEAE
}

\section{CAN THE CO-OCURRENCE OF PLANTS IN CAATINGA BE EXPLAINED BY THE FACILITATION PROCESS? A CASE STUDY OF TWO SPECIES OF FABACEA}

\author{
Djane Ventura de Azevedo ${ }^{1}$ Roberta Boscaini Zandavalli ${ }^{2}$ Tiago Osório Ferreira ${ }^{3}$ \\ Fernando Roberto Martins ${ }^{4}$ Francisca Soares de Araújo ${ }^{5}$
}

\begin{abstract}
RESUMO
Diferenças heteroespecíficas nas taxas de crescimento em altura, na produção de biomassa e na área foliar entre espécies coocorrentes são formas de medir a ocorrência de interações: facilitação ou competição. Com base no conceito mais amplo de facilitação, este tipo de interação positiva tem sido relatado para explicar a coocorrência de espécies em ambientes com alta limitação de recurso, a exemplo de climas árticos e áridos. O objetivo deste estudo foi avaliar se a facilitação pode favorecer o desenvolvimento inicial das populações de Mimosa caesalpiniifolia e Bauhinia cheilantha, duas espécies típicas da região da Caatinga, em casa de vegetação. As plântulas de Mimosa caesalpiniifolia Benth e de Bauhinia cheilantha (Bongard) foram cultivadas em vasos por 120 dias, nas proporções de $4: 1 ; 3: 2 ; 2: 3 ; 1: 4 ; 5: 0 ; 0: 5$. Também foram realizadas avaliações na estrutura e em alguns teores (nitrogênio e fósforo) de nutrientes no solo e na folha das plântulas. Os resultados de crescimento, alocação de biomassa e concentrações de nitrogênio e fósforo nas folhas de Mimosa caesalpiniifolia foram influenciados positivamente com o aumento da densidade de indivíduos de Bauhinia cheilantha (Bongard) Steudel. No entanto, esta última espécie teve a menor taxa de crescimento de competição intraespecífica. Se for adotado o conceito mais amplo de facilitação, pode-se afirmar que houve interações positivas. Por se tratar de duas espécies de Fabaceae que podem apresentar associações com fungos micorrízicos e com bactérias diazotróficas, o principal nutriente limitante para o bom desempenho na coocorrência destas duas populações deverá ser o fósforo, pois não houve diferenças na quantidade de nitrogênio disponível no solo entre o início e o final do experimento.
\end{abstract}

Palavras-chave: interações; intraespecíficas; interespecíficas; desenvolvimento inicial de plantas.

\begin{abstract}
Heteroespecific differences in growth rates in height, biomass production and leaf area among co-occurring species are ways of measuring the occurrence of interaction: facilitation or competition. Based on the broader concept of facilitation, this type of positive interaction has been reported to explain the co-occurrence of species in environments with high resource limitations, such as arctic and arid climates. The objective of this study was to evaluate if facilitation could favor the initial development of Mimosa caesalpinlifolia and Bauhinia cheilantha populations, two species typical of the Caatinga region, under greenhouse conditions. The seedlings of Mimosa caesapinifolia Benth and Bauhinia cheilantha (Bongard) were cultivated in pots for 120 days in the ratios of $4: 1 ; 3: 2 ; 2: 3 ; 1: 4 ; 5: 0 ; 0: 5$. Evaluations about structure and of some nutrient

1 Bióloga, MSc., Professora do Centro de Educação, Ciências e Tecnologia da Universidade Estadual do Ceará, Rua Sólon Medeiros, s/n, CEP 60000-000, Tauá (CE), Brasil. djane.azevedo@uece.br

2 Bióloga, Dr ${ }^{\mathrm{a}}$., Professora do Departamento de biologia, Centro de ciências da Universidade Federal do Ceará, Av. Humberto Monte, s/n, CEP 60455-760, Fortaleza (CE), Brasil. zandavalli@yahoo.com.br

3 Engenheiro Agrônomo, Dr., Professor da Escola Superior de Agricultura Luiz Queiroz da Universidade de São Paulo, Av. Pádua Dias, 11, CEP 13418-260, Piracicaba (SP), Brasil. tiago@ufc.br

4 Biólogo, Dr., Professor do Departamento de Biologia Vegetal da Universidade Estadual de Campinas, Rua Monteiro Lobato, 255, CEP13083-970, Campinas (SP), Brasil. fmartins@unicamp.br

5 Bióloga, Dr ${ }^{\text {a }}$, Professora do Departamento de Biologia, Centro de Ciências da Universidade Federal do Ceará, Av. Humberto Monte, s/n, CEP 60455-760, Fortaleza (CE), Brasil. tchesca@ufc.br
\end{abstract}

Recebido para publicação em 4/02/2014 e aceito em 6/11/2017

Ci. Fl., v. 28, n. 4, out. - dez., 2018 
contents (nitrogen and phosphorus) in the soil and leaf of the seedlings. The results of growth, biomass allocation and concentrations of nitrogen and phosphorus in leaves of Mimosa caesalpinlifolia were positively influenced by the increase of the density of Bauhinia cheilantha (Bongard) Steudel individuals. However, the latter species had the lowest growth rate of intra-specific competition. If we adopt the broadest concept of facilitation, we can affirm that there were positive interactions. As both are species of Fabaceae that may present associations with mycorrhizal fungi and diazotrophic bacteria, the main limiting nutrient for good performance in the co-occurrence of these two populations should be phosphorus, since there were no differences in the amount of nitrogen available in the soil between the beginning and the end of the experiment. Keywords: interactions; intraespecific; interspecific; initial plant development.

\section{INTRODUÇÃO}

A diferença de crescimento em altura, produção de biomassa e área foliar são formas de medir a ocorrência de competição (BLOOR; LEADLEY; BARTHES, 2007) ou facilitação (BANUET; VERDÚ, 2008) entre plantas. A espécie facilitada apresentará maior crescimento na presença da facilitadora se comparado com o seu crescimento isoladamente (FORRESTER; VANCLAY; FORRESTER, 2011).

A ocorrência de facilitação entre espécies já é bastante conhecida na literatura (TIRADO; PUGNAIRE, 2005). Os efeitos da relação de facilitação podem ser positivos para ambas as espécies (BANUET; VERDÚ, 2008) ou negativos para um dos indivíduos envolvidos (CALLAWAY, 2007). Em ambientes sob climas árticos, os efeitos negativos dos ventos fortes e das temperaturas extremas são atenuados pela presença de plantas que aumentam a umidade do solo abaixo de sua copa (CAVIERES et al., 2006). Em ambientes áridos, o sombreamento e o aporte de serrapilheira sob as copas minimizam os extremos de temperatura e a escassez hídrica, que é o principal fator limitante (SCHWINNING, 2004). No entanto, o aumento da demanda dos indivíduos por recursos substitui a facilitação inicial pela competição (CHESSON et al., 2004; STHULTZ; GEHRING; WHITHAN, 2007; ARMAS; PUGNAIRE, 2009; NUÑES et al., 2009).

A resposta de indivíduos à competição interespecífica por nutrientes tem sido demonstrada, de forma geral, nas associações entre herbáceas e plântulas de espécies arbóreas (BLOOR; LEADLEY; BARTHES, 2007; VAN DER WAAL et al., 2009) ou entre indivíduos de espécies arbóreas em diferentes estágios ontogenéticos (LEWIS; TANNER, 2000). Segundo Paine Timothy et al. (2008), a inexistência de competição por nutrientes entre plântulas em florestas neotropicais está relacionada à baixa densidade de indivíduos. Já para Klironomos (2002), associações positivas explicam a ocorrência de plântulas em altas densidades.

Leguminosas beneficiadas por associações com micorrizas e/ou bactérias diazotróficas facilitam o crescimento de indivíduos de outras espécies (FREITAS et al., 2010) em sua vizinhança, através da oferta de água e nutrientes (SCHWINNING; SALA, 2004; ZHAO et al., 2007; FORRESTER; VANCLAY; FORRESTER, 2011), permitindo o investimento em crescimento vegetativo por parte das plantas facilitadas (MANGOLD et al., 2004; CHEAIB et al., 2005; ZHAO et al., 2007; RICHARDSON; BAREA; MCNEILL, 2009).

A coocorrência de plântulas heteroespecíficas que utilizam recursos similares, como água e nutrientes, aumenta a competição interespecífica. Por outro lado, a facilitação será a interação ocorrente, quando houver diferenças na demanda por recursos entre as espécies. Para testar essas previsões, foram selecionadas duas espécies de Leguminosae: Mimosa caesalpiniifolia Benth e Bauhinia cheilantha (Bongard) Steud que coocorrem com altas densidades em áreas de Caatinga do estado do Ceará (COSTA; ARAUJO, 2012). Para avaliar qual das espécies é facilitada e facilitadora, foram considerados: 1) que a espécie facilitada apresente altura, biomassa e área foliar superiores à espécie facilitadora; 2) que a espécie facilitada apresente competição intraespecífica baixa. Desta forma, o estudo mostra como a coocorrência de plântulas de espécies arbóreas aumenta as chances de estabelecimento dos indivíduos, podendo atuar como uma das forças estruturadoras da comunidade vegetal. 


\section{MATERIAL E MÉTODO}

\section{Espécies-alvo}

As duas espécies selecionadas para este estudo, Bauhinia cheilantha (Bongard) Steud e Mimosa caesalpiniifolia Benth, coocorrem com altas densidades em áreas de caatinga do estado do Ceará (COSTA; ARAUJO, 2012). Sementes dessas duas espécies foram coletadas da Caatinga na Reserva Particular do

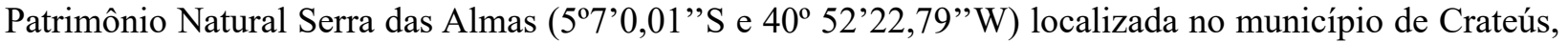
centro-oeste do estado do Ceará, na região nordeste do Brasil (COSTA; ARAUJO, 2012).

\section{Condições de cultivo e delineamento experimental}

Para verificar que tipos de interações intraespecífica e interespecífica predominam no desenvolvimento inicial das duas espécies de Fabaceae coocorrentes na Caatinga, analisaram-se a alocação de biomassa, o crescimento e a absorção de nutrientes durante o desenvolvimento inicial em experimentos em casa de vegetação, sob condições naturais de temperatura. Durante o período de experimentação, a temperatura média diária foi de $30^{\circ} \mathrm{C}$ (médias máximas de $31^{\circ} \mathrm{C}$ com médias das mínimas de $23,4^{\circ} \mathrm{C}$ ), e a umidade relativa do ar variou de $91 \%$ (máxima) a 70,3\% (mínima). Sementes de Bauhinia cheilantha e Mimosa caesalpiniifolia foram semeadas em bandejas de isopor previamente preenchidas com solo retirado da área de ocorrência natural destas espécies. Três semanas após o início da germinação, foram selecionadas plântulas com alturas similares de cada uma das espécies. Em seguida, os indivíduos de cada espécie foram transferidos para vasos circulares de $25 \mathrm{~cm}$ de diâmetro por $20 \mathrm{~cm}$ de profundidade, previamente preenchidos com solo proveniente do local de origem das sementes, coletado na profundidade de $0-10 \mathrm{~cm}$. Cada vaso foi irrigado diariamente até a capacidade do vaso sem que nenhum suplemento mineral fosse adicionado.

O delineamento experimental foi inteiramente casualizado em esquema fatorial $(2 \times 5)$ com duas espécies e cinco proporções para cada espécie. Foram transplantadas para vasos nas proporções de 4 indivíduos de Mimosa caesalpiniifolia e 1 indivíduo de Bauhinia cheilantha; 3 de Mimosa caesalpiniifolia e 2 de Bauhinia cheilantha; 2 de Mimosa caesalpiniifolia e 3 de Bauhinia cheilantha, 1 de Mimosa caesalpiniifolia e 4 de Bauhinia cheilantha, 5 indivíduos de Mimosa caesalpiniifolia e 5 de Bauhinia cheilantha, com quatro repetições para cada tratamento de frequência.

O pequeno número de repetições utilizado neste experimento se deve à baixa produção de sementes em resposta à ocorrência de estação chuvosa irregular dos últimos três anos. Além disso, as sementes coletadas apresentaram taxas de germinação abaixo do comumente esperado, por isso, somente foram possíveis quatro repetições por tratamento de frequência. Para avaliar o crescimento dos indivíduos de ambas as espécies na ausência de vizinhos, transferiu-se uma plântula de cada espécie para vasos separados e avaliaram-se as respostas de crescimento de cada espécie sem efeitos de vizinho.

\section{Coleta de dados}

Para verificar a alocação de biomassa de cada espécie, ao final de 120 dias de experimento, foi separada a parte aérea (caules e folhas) de cada indivíduo de cada tratamento. Foi delimitado um período de observação de 120 dias por que experimentos em vasos por período superior podem impedir o crescimento das plantas devido à inexistência de espaço para desenvolvimento radicular. O sistema radicular foi retirado do vaso e lavado cuidadosamente para evitar a perda de raízes. As amostras de folhas, caule e raízes foram desidratadas a $65^{\circ} \mathrm{C}$ por 48 horas e posteriormente pesadas para a obtenção da biomassa seca acumulada (EMBRAPA, 2005). A limitação por nutrientes na expansão foliar das espécies foi determinada por meio de um medidor de área foliar Li-Cor, modelo LI-3100 (Lincoln, Nebraska, USA). Para medir o comprimento da parte aérea, uma fita métrica foi disposta da superfície do solo até o primórdio foliar localizado no ápice da planta. O diâmetro do caule foi obtido com a utilização de paquímetro digital, e as mensurações foram realizadas sempre na mesma posição no caule.

Para avaliar se houve competição por nutrientes, amostras do solo utilizados nos vasos para cres- 
cimento das plantas foram previamente caracterizadas quanto à granulometria ( $77 \%$ areia, $19 \%$ silte e $4 \%$ argila), concentração de nitrogênio $(0,98 \mathrm{~g} / \mathrm{kg})$, de fósforo $(23,9 \mathrm{mg} / \mathrm{kg})$ e de matéria orgânica $(14,41 \mathrm{~g} / \mathrm{kg})$. No final do experimento, as amostras do solo provenientes dos vasos de cada tratamento foram desidratadas, destorroadas, peneiradas em malha de $2 \mathrm{~mm}$ e analisadas para a determinação das concentrações de nitrogênio e de fósforo remanescente.

Para avaliar as respostas nutricionais da planta à limitação de nutrientes, todas as folhas jovens e totalmente expandidas de cada indivíduo das espécies de Bauhinia cheilantha e de Mimosa caesalpiniifolia foram selecionadas. Tais folhas foram desidratadas por 48 horas a $60^{\circ} \mathrm{C}$ e moídas para posterior análise dos teores de nitrogênio e fósforo. Para extração de nitrogênio, foi utilizada a digestão sulfúrica, enquanto o fósforo foi extraído a partir da digestão nitroperclórica. As concentrações de nitrogênio foram obtidas a partir do método de Kjeldahl, enquanto o fósforo foi determinado por meio do método colorimétrico do azul de molibdênio (EMBRAPA, 1997).

\section{Análise de dados}

A ANOVA de dois fatores foi utilizada para testar a interação entre a espécie (primeiro fator) e a proporção de indivíduos (segundo fator) (BLANCK, 2010). Nos casos em que foi demonstrado haver interação significativa entre os fatores, utilizou-se o teste de Tukey para verificar se as médias das variáveis respostas (biomassa seca, área foliar especifica, altura, teores de nitrogênio e fósforo) difeririam entre os diferentes tratamentos. Para verificar o desenvolvimento de cada espécie sem a influência da proporção heteroespecífica ou monoespecífica, compararam-se as médias dos resultados obtidos para o controle, indivíduos que cresceram isoladamente, com o dos tratamentos de diferentes densidades, por meio do Teste de Dunnett.

\section{RESULTADOS E DISCUSSÃO}

\section{Crescimento das espécies em isolamento: competição e facilitação}

Os valores médios de altura ( $F: 8,08 \mathrm{p}<0,001)$, área foliar ( $\mathrm{F}: 27,57 \mathrm{p}<0,001)$, biomassa foliar $(\mathrm{F}$ : $14,39 \mathrm{p}<0,001$ ), biomassa caulinar ( $\mathrm{F}: 16,59 \mathrm{p}<0,001)$ e biomassa radicular $(\mathrm{F}: 3,92 \mathrm{p}<0,005)$ de Mimosa caesalpiniifolia foram menores em crescimentos isolados. Por outro lado, os indivíduos de Bauhinia cheilantha crescidos em isolamento (controle) apresentaram maiores desenvolvimentos em altura (F: 35,87 $\mathrm{p}<0,001$ ), área foliar (F: 191,89 p<0,001), biomassa foliar (F: 28,24 p<0,001), biomassa caulinar (F: 182,13 $\mathrm{p}<0,001)$ e biomassa radicular (F: $15,72 \mathrm{p}<0,001)$ quando comparados aos indivíduos crescidos na presença de vizinhos (Tratamentos heteroespecíficos e monoespecíficos, Tabela 1).

TABELA 1: Crescimento e alocação de biomassa de indivíduos (controle) e de tratamentos de proporção de indivíduos de Mimosa caesalpiniifolia (M) e Bauhinia cheilantha (B).

TABLE 1: Growth and biomass allocation of individuals (control) and dosage treatments of Mimosa caesalpiniifolia (M) and Bauhinia cheilantha (B).

\begin{tabular}{|c|c|c|c|c|c|}
\hline Tratamentos & Altura & Área foliar & Biomassa foliar & Biomassa caulinar & Biomassa radicular \\
\hline & $\mathrm{cm}$ & $\mathrm{cm}^{2}$ & 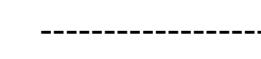 & ---------g- & ------------ \\
\hline & \multicolumn{5}{|c|}{ Mimosa caesalpiniifolia } \\
\hline Controle & 59,57 & 1860 & 4,2 & 3,54 & 0,44 \\
\hline $1 \mathrm{M}-4 \mathrm{~B}$ & $108,33^{*}$ & $3497,80^{*}$ & $15,23^{*}$ & $11,18^{*}$ & $1,65^{*}$ \\
\hline $2 \mathrm{M}-3 \mathrm{~B}$ & $95,32^{*}$ & $1945,88^{\mathrm{ns}}$ & $8,72^{\mathrm{ns}}$ & $7,04^{\mathrm{ns}}$ & $1,71^{*}$ \\
\hline $3 \mathrm{M}-2 \mathrm{~B}$ & $51,95^{\mathrm{ns}}$ & $1722,51^{\mathrm{ns}}$ & $5,90^{\mathrm{ns}}$ & $12,51^{*}$ & $0,94^{\mathrm{ns}}$ \\
\hline
\end{tabular}


TABELA 1: Continuação...

TABLE 1: Continued...

\begin{tabular}{|c|c|c|c|c|c|}
\hline Tratamentos & Altura & Área foliar & Biomassa foliar & Biomassa caulinar & Biomassa radicular \\
\hline & $\mathrm{cm}$ & $\mathrm{cm}^{2}$ & \multicolumn{3}{|c|}{ 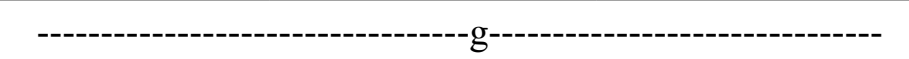 } \\
\hline $4 \mathrm{M}-1 \mathrm{~B}$ & $77,98^{\mathrm{ns}}$ & $1167,94^{*}$ & $5,38^{\mathrm{ns}}$ & $4,12^{\mathrm{ns}}$ & $1,20^{\mathrm{ns}}$ \\
\hline \multirow[t]{2}{*}{$5 \mathrm{M}-0 \mathrm{~B}$} & $75,31^{\mathrm{ns}}$ & $1245,03^{\mathrm{ns}}$ & $4,26^{\mathrm{ns}}$ & $3,41^{\mathrm{ns}}$ & $0,90^{\mathrm{ns}}$ \\
\hline & \multicolumn{5}{|c|}{ Bauhinia cheilantha } \\
\hline Controle & 78,83 & 2128,74 & 7,83 & 6,6 & 7,32 \\
\hline $1 \mathrm{~B}-4 \mathrm{M}$ & $26,02^{*}$ & $139,22^{*}$ & $0,64^{*}$ & $1,03^{*}$ & $1,03^{*}$ \\
\hline $2 \mathrm{~B}-3 \mathrm{M}$ & $23,07^{*}$ & $283,00^{*}$ & $0,71^{*}$ & $0,69^{*}$ & $1,03^{*}$ \\
\hline $3 \mathrm{~B}-2 \mathrm{M}$ & $20,88^{*}$ & $292,60^{*}$ & $0,64^{*}$ & $0,73^{*}$ & $1,27^{*}$ \\
\hline $4 \mathrm{~B}-1 \mathrm{M}$ & $24,13^{*}$ & $383,13^{*}$ & $1,00^{*}$ & $1,14^{*}$ & $0,98^{*}$ \\
\hline $5 \mathrm{~B}-0 \mathrm{M}$ & $29,97^{*}$ & $426,81^{*}$ & $1,22^{*}$ & $1,59^{*}$ & $1,07^{*}$ \\
\hline
\end{tabular}

*Médias diferem significativamente do controle na mesma coluna pelo teste de Dunnett $(\mathrm{p}>0,05)$; $\mathrm{ns}=$ diferença não significativa.

A comparação entre as médias de crescimento em altura, biomassa seca e área foliar dos indivíduos de Mimosa caesalpiniifolia nos tratamentos de proporção monoespecífico (Tabela 1, 5M-0B) e controle não mostrou diferenças significativas quando comparadas ao desenvolvimento dos indivíduos em isolamento. Portanto, os valores de crescimento dos indivíduos de Mimosa caesalpiniifolia nos vasos de maior proporção não se deve à ocorrência de competição intraespecífica, mas sim à redução da proporção de indivíduos de Bauhinia cheilantha. Embora seja esperado que a competição intraespecífica se intensifique com o aumento da densidade de indivíduos coespecíficos (FETENE, 2003), conforme Carvalho, Alves e Matins (2011), as espécies apresentam diferenças na demanda de recursos para crescimento, de modo que algumas necessitam de mais água e nutrientes, enquanto outras requerem menos recursos para investir em crescimento vegetativo. No entanto, as médias de altura, área foliar e alocação de biomassa observadas para Bauhinia cheilantha entre os tratamentos de proporção intraespecífico (Tabela 1,5B-0M) foram inferiores quando comparadas ao controle. A similaridade na redução das médias de crescimento e alocação de biomassa em Bauhinia cheilantha nos tratamentos coespecífico (5B-0M) e heteroespecíficos (Figura 1) indicam que essa espécie apresenta intolerância a vizinhos coespecíficos e heteroespecíficos. Taxas de crescimento em altura, biomassa e área foliar menores indicam a ocorrência de competição (BLOOR; LEADLEY; BARTHES, 2007), o que pode permitir que uma das espécies envolvidas na interação tenha o seu crescimento facilitado em detrimento do crescimento de outra (FORRESTER; VANCLAY; FORRESTER, 2011).

Diferenças de tamanho inicial dos indivíduos afeta a natureza das interações biológicas (NUÑES et al., 2009). Contudo, os tamanhos encontrados no final do experimento para indivíduos crescidos em isolamento mostram que as duas espécies apresentam similaridade na altura.

\section{Proporção de indivíduos: efeito facilitador}

As variáveis de biomassa foliar $(\mathrm{F}: 15,4 ; \mathrm{p}<0,01)$, caulinar $(\mathrm{F}: 24,91 ; \mathrm{p}<0,01)$, altura $(\mathrm{F}: 61,36$; $\mathrm{p}<0,01)$ e área foliar $(\mathrm{F}: 95,5 ; \mathrm{p}<0,01)$ para a interação entre os fatores de proporção de indivíduos e espécie demonstram que: 1) na presença de outros indivíduos de Bauhinia cheilantha, o crescimento em altura e alocação de biomassa foram superiores em Mimosa caesalpiniifolia (proporções de 1:4 e 2:3) (Figura 1). Esses resultados indicam a ocorrência de efeito positivo sobre Mimosa caesalpiniifolia e negativo para a espécie facilitadora. Para Callaway (2007), as interações positivas ocorrem mesmo quando existe um efeito negativo para a espécie facilitadora, desde que, conforme Sthultz, Gehring e Whithan (2007), isso seja 
registrado para indivíduos na mesma fase de desenvolvimento. Nestes casos, a intensidade da competição interespecífica entre plântulas é considerada baixa, e as espécies que apresentam competição intraespecífica apenas sobre grandes densidades se beneficiam da proximidade com espécies facilitadoras (PAINE TIMOTHY et al., 2008; CARVALHO; ALVES; MATINS, 2011).
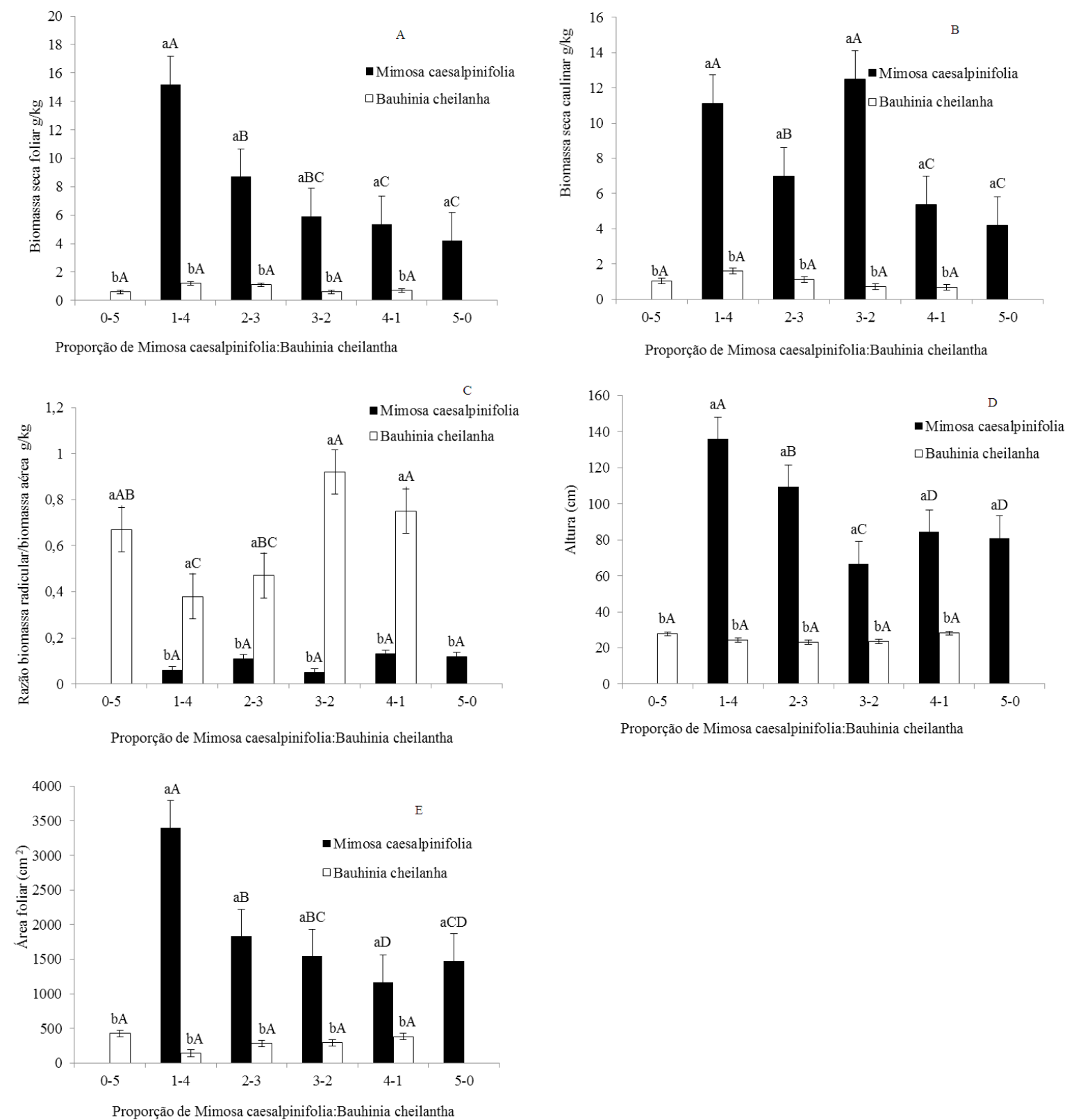

Letras minúsculas distintas representam diferenças estatísticas entre espécies, e letras maiúsculas distintas representam diferenças estatísticas entre frequências a $5 \%$ de probabilidade.

FIGURA 1: Alocação de biomassa foliar (A); caulinar (B); razão biomassa radicularbiomassa aérea (C); altura do caule (D) e área foliar (E) de Mimosa caesalpiniifolia e Bauhinia cheilantha em frequências de 1:4; $2: 3 ; 3: 2 ; 4: 1 ; 5: 0$.

FIGURE 1: Allocation of foliar biomass (A), aerial biomass (B); Root biomass ratio / aerial (C); stem height (D) and leaf area (E) of Mimosa caesalpiniifolia and Bauhinia cheilantha at frequencies of 1:4, 2:3, 3:2, $4: 1,5: 0$. 


\section{Concentração de nitrogênio e fósforo}

As análises de solo realizadas nas amostras obtidas de cada um dos cinco tratamentos de frequência não revelam diferenças significativas na disponibilidade de nitrogênio para o crescimento das espécies, entre a amostra inicial de $0,98 \mathrm{~g} / \mathrm{kg}(\mathrm{F}: 1,49 ; \mathrm{p}>0,05)$ e as amostras de solo coletadas após a retirada das plantas (Tabela 2). Os dados apontam que o nitrogênio não foi um elemento limitante para o crescimento dessas espécies, embora a baixa disponibilidade de nitrogênio reduza o crescimento e interfira na biomassa aérea das plantas (MANGOLD et al., 2004). As associações entre leguminosas e bactérias diazotróficas aumentam a absorção de nitrogênio para as plantas (FREITAS et al., 2010).

A redução nas concentrações de fósforo encontradas entre a amostra inicial de 23,9 mg/ kg (F:6,95; $\mathrm{p}<0,01$ ) e os tratamentos com diferentes proporções de indivíduos revelam diferenças significativas (nas frequências de 2, 4 e 5 indivíduos de Mimosa caesalpiniifolia, Tabela 2). A menor razão entre biomassa radicular e aérea em Mimosa caesalpiniifolia ocorre nas proporções de 1-4 e 2-3 (Figura 1C), o que pode estar relacionado ao efeito facilitador de Bauhinia cheilantha, pois a maior razão entre biomassa radicular e biomassa aérea desta espécie ocorreu em resposta ao aumento da densidade de indivíduos de Mimosa caesalpiniifolia (Figura 1C; tratamento 3-2 e 4:1) e aponta para um maior investimento na parte aérea.

TABELA 2: Concentrações de nitrogênio e fósforo no solo coletado dos vasos ao final do experimento. Tratamentos de frequência (M) Mimosa caesalpiniifolia e (B) Bauhinia cheilantha.

TABLE 2: Concentrations of nitrogen and phosphorus in soil collected from pots at the end of the experiment. Frequency treatements (M) Mimosa caesalpiniifolia and (B) Bauhinia cheilantha.

\begin{tabular}{cccc}
\hline Espécies & Frequência de indivíduos por vaso & Nitrogênio & Fósforo \\
\hline & & $(\mathrm{g} / \mathrm{kg})$ & $(\mathrm{mg} / \mathrm{kg})$ \\
\hline \multirow{3}{*}{ Bauhinia cheilantha $(\mathrm{B})$} & 1M-4B & $0,75 \pm 0,08^{\mathrm{ns}}$ & $20,01 \pm 1,69^{\mathrm{ns}}$ \\
Mimosa caesalpiniifolia $(\mathrm{M})$ & 2M-3B & $0,73 \pm 0,07^{\mathrm{ns}}$ & $15,19 \pm 1,48^{*}$ \\
& 4M-1B & $0,7 \pm 0,1^{\mathrm{ns}}$ & $16,46 \pm 0,98^{*}$ \\
& 5M-0B & $1,04 \pm 0,42^{\mathrm{ns}}$ & $15,58 \pm 2,89^{*}$ \\
& 0M-5B & $0,76 \pm 0,05^{\mathrm{ns}}$ & $19,20 \pm 1,70{ }^{\text {ns }}$ \\
& Controle & $0,71 \pm 0,05$ & $23,90 \pm 1,53$ \\
\hline
\end{tabular}

*Médias diferem significativamente do controle na mesma coluna pelo teste de Dunnett $(\mathrm{p}>0,05)$; ns = diferenças não significativa.

Embora a espécie Bauhinia cheilantha apresente concentrações superiores de fósforo por miligrama de tecido foliar (F: 16,51 p < 0,01), o acúmulo de fósforo por planta nesta espécie foi baixo em todos os tratamentos de densidade, devido aos baixos valores de biomassa apresentado pelos indivíduos. (Tabela 3). Isso significa que parte do fósforo absorvido foi mobilizado para o crescimento, pois espécies mais eficientes na absorção desse elemento podem investir em alocação de biomassa aérea e biomassa radicular (ALLCOCK, 2002). Esses argumentos explicam o efeito positivo da presença de Bauhinia cheilantha sobre o crescimento de Mimosa caesalpiniifolia. 
TABELA 3: Teores e acúmulo de fósforo em Mimosa caesalpiniifolia e Bauhinia cheilantha. Tratamentos de Frequências (M) Mimosa caesalpiniifolia e (B) Bauhinia cheilantha.

TABLE 3: Levels and phosphorus accumulation in Mimosa caesalpiniifolia and Bauhinia cheilantha. Frequency treatments (M) Mimosa caesalpiniifolia and (B) Bauhinia cheilantha.

\begin{tabular}{|c|c|c|c|c|}
\hline \multirow[b]{2}{*}{ Densidades } & \multicolumn{2}{|c|}{ Teores de Fósforo por mg de folha } & \multicolumn{2}{|c|}{ Acúmulo de Fósforo por planta } \\
\hline & $\begin{array}{c}\text { Mimosa } \\
\text { caesalpiniifolia }(M)\end{array}$ & $\begin{array}{c}\text { Bauhinia } \\
\text { cheilantha }(B)\end{array}$ & $\begin{array}{c}\text { Mimosa } \\
\text { caesalpiniifolia }(M)\end{array}$ & $\begin{array}{c}\text { Bauhinia } \\
\text { cheilantha }(B)\end{array}$ \\
\hline & \multicolumn{2}{|c|}{$\mathrm{mg} \mathrm{kg}^{-1}$} & \multicolumn{2}{|c|}{ mg planata $^{-1}$} \\
\hline $1 \mathrm{M}-4 \mathrm{~B}$ & $143,27 \mathrm{bcA}$ & $249,67 \mathrm{cA}$ & $2,17 \mathrm{aA}$ & $0,15 \mathrm{bA}$ \\
\hline $2 \mathrm{M}-3 \mathrm{~B}$ & $119,24 \mathrm{cA}$ & $278,62 \mathrm{cA}$ & $1,03 \mathrm{aB}$ & $0,17 \mathrm{bA}$ \\
\hline $3 \mathrm{M}-2 \mathrm{~B}$ & $111,08 \mathrm{bB}$ & $249,24 \mathrm{bA}$ & $0,65 \mathrm{aBC}$ & $0,16 \mathrm{bA}$ \\
\hline $4 \mathrm{M}-1 \mathrm{~B}$ & $104,52 \mathrm{aA}$ & $267,25 \mathrm{bB}$ & $0,56 \mathrm{aC}$ & $0,26 \mathrm{aA}$ \\
\hline $5 \mathrm{M}-0 \mathrm{~B}$ & $153,36 \mathrm{aB}$ & $212,24 \mathrm{aA}$ & $0,653 \mathrm{aBC}$ & $0,33 \mathrm{aA}$ \\
\hline
\end{tabular}

Letras minúsculas distintas representam diferenças estatísticas entre indivíduos, e letras maiúsculas distintas representam diferenças estatísticas entre espécies a $5 \%$ de probabilidade.

\section{CONCLUSÃO}

Ficou evidenciado que apenas Mimosa caesalpiniifolia foi facilitada. Os teores superiores de fósforo nos tecidos de Bauhinia cheilantha, quando comparados à Mimosa caesalpiniifolia, demonstraram que a primeira espécie apresenta maior demanda para este nutriente. É possível que, na proximidade da área de absorção radicular de Bauhinia cheilantha, Mimosa caesalpiniifolia seja beneficiada através da aquisição de fósforo. Serão necessários mais estudos que possam verificar as relações entre as concentrações de fósforo de Bauhinia cheilantha e aspectos de sua arquitetura radicular, existência de associações com micorrizas arbusculares e efeitos dessa associação sobre a arquitetura radicular de Bauhinia cheilantha.

\section{AGRADECIMENTOS}

Os autores agradecemos à FUNCAP, pela bolsa de doutorado concedida a primeira autora; à Universidade Estadual do Ceará (UECE); aos professores, técnicos e bolsistas do laboratório de pedologia do Departamento de Solos da Universidade Federal do Ceará; ao CNPq, pelas bolsas de Produtividade em Pesquisa.

\section{REFERÊNCIAS}

ALLCOCK, K. G. Effects of phosphorus on growth and competitive interactions of native and introduced species found in white box woodlands. Austral Ecology, Carlton, v. 27, p. 638-646, 2002.

ARMAS, C.; PUGNAIRE, F. I. Ontogenetic shifts in interactions of two dominant shrub species in a semi-arid coastal sand dune system. Journal of Vegetation Science, Knivsta, v. 20, p. 535-546, 2009.

BANUET, A.V.; VERDÚ, M. Temporal shifts from facilitation to competition occur between closely related taxa. Journal of Ecology, Oxford, v. 96, p. 489-494, 2008.

BLANCK, R. R. Intraespecific and interspecific pair-wise seedling competition between exotic annual grasses and native perennials: plant and soil relationship. Plant and Soil, The Hage, v. 326, p. 331-343, 2010.

BLOOR, J. M. G.; LEADLEY, P. W.; BARTHES, L. Responses of Fraxinus excelcior seedlings to grass induced above- and below-ground competition. Plant Ecology, Dordrecht, v. 194, p. 293-304, 2007.

CALLAWAY, R. M. Positive interactions and interdependence in plant communities. USA: Springer, 2007. $413 \mathrm{p}$.

CARVALHO, L. B.; ALVES, P. L. C. A.; MATINS, J. V. F. Effects of plant density and proportion on the 
interactions between wheat with alexander grass plants. Bragantia, Campinas, v. 70, p. 40-45, 2011. CAVIERES, L. A. et al. Positive interactions between alpine plants species and the nurse cushion plant Laretia acaulis do no increase with elevation in the Andes of central Chile. New Phytologist, Cambridge, v. 169, p. 59-70, 2006.

CHEAIB, A. et al. Interacive effects of phosphorus and light availability on early growth of maritime pine seedlings. Annals of Forest Science, Les Ulis, v. 62, p. 575-583, 2005.

CHESSON, P. et al. Resource pulses, species interactions, and diverse maintenance in arid and semiarid environment. Oecologia, Berlin, v. 141, p. 236-253, 2004.

COSTA, R. C.; ARAUJO, F. S. Physionomy and structure of a Caatinga with Cordiaoncocalix (Boraginaceae), a new type of community in Andrade-Limás classification of caatingas. Rodriguesia, Rio de Janeiro, v. 63, p. 269-273, 2012.

EMBRAPA. Manual de métodos de análises de solo. 2. ed. Rio de Janeiro: EMBRAPA, 1997. 212 p. EMBRAPA. Solo, água, nutrição vegetal, nutrição animal e alimentos. 2. ed. São Carlos: EMBRAPA, 2005. $212 \mathrm{p}$.

FETENE, M. Intra and inter-specific competition between seedlings of Acacia etbaica and a perennial grass (Hyparrenia hirta). Journal of Arid Environment, [s. 1.], v. 55, p. 441-451, 2003.

FORRESTER, D. I.; VANCLAY, J. K.; FORRESTER, R. I. The balance between facilitation and competition in mixtures of eucalyptus and Acacia changes as stand develop. Oecologia, Berlin, v. 166, p. 265-272, 2011.

FREITAS, A. D. S. et al. Biological nitrogen fixation in tree legumes of the Brasilian semi-arid Caatinga. Journal of Arid Environment, [s. 1.], v. 74, p. 344-349, 2010.

KLIRONOMOS, J. N. Feedback with soil biota contributes to plant rarity and invasiveness in communities. Nature, London, v. 417, p. 67-70, 2002.

LEWIS, S. L.; TANNER, E. V. J. Effects of above and belowground competition on growth and survival of rain forest tree seedlings. Ecology, Brooklyn, v. 81, p. 2525-2538, 2000.

MANGOLD, J. K. et al. Identification of limiting resource within a semi-arid plant association. Journal of Arid environments, [s. 1.], v. 58, p. 309-320, 2004.

NUÑEZ, C. I. et al. When do nurse plants stop nursing? Temporal changes in water stress levels in Austrocedrus chilensis growing within and outside shrubs. Journal of Vegetation Science, Knivsta, v. 20, p. 1064-1071, 2009.

PAINE TIMOTHY, C. E. et al. Weak competition among tropical tree seedlings: implications for species coexistence. Biotropica, Washington, v. 40, p. 432-440, 2008.

RICHARDSON, A. E.; BAREA, J.-M.; MCNEILL, A. M. Acquisition of phosphorus and nitrogen in the rhizosphere and plant growth promotion by microorganisms. Plant Soil, [s. 1.], v. 321, p. 305-339, 2009.

SCHWINNING, S. et al. Thresholds, memory, and sazonality: understand pulse dynamics in arid/semi-arid ecosystens. Oecologia, Berlin, v. 141, p. 191-193, 2004.

SCHWINNING, S; SALA, O. E. Hierarchy of responses to resource pulses in arid and semiarid ecosystems. Oecologia, Berlin, v. 141, p. 211-220, 2004.

STHUlTZ, C. M.; GEHRING, C. A.; WHITHAN, T. G. Shifts from competition to facilitation between a foundation tree and a pioneer shrub across spatial and temporal scales in a semiarid woodland. New Phytologist, Cambridge, v. 173, p. 135-145, 2007.

TIRADO, R.; PUGNAIRE, F. I. Community structure and positive interactions in constraining environments. Oikos, Copenhagen, v. 111, p. 437-444, 2005.

VAN DER WALL, C. et al. Water and nutrients alter herbaceous competitive effects on tree seedlings in a semiarid savanna. Journal of ecology, Oxford, v. 97, p. 430-439, 2009.

ZHAO, H.-L. et al. Shrub facilitation of desert land restoration in the Horqin Sand Land of inner Mongolia. Ecological Engineering, Oxford, v. 31, p. 1-8, 2007. 